\title{
The Modes of Future China Electricity Markets and Its Impacts on IT Systems of System Operator
}

\author{
Yuandong $\mathrm{MA}^{1,}$, , Zhenglin Yang ${ }^{2, \mathrm{~b}}$ \\ ${ }^{1}$ No.8 NARI road, Nanjing, Jiangsu, China, 210003 \\ amayuandong@sgepri.sgcc.com.cn, byangzhenglin@epri.sgcc.com.cn
}

\begin{abstract}
Keywords: electricity market, electric power system reform, system operator, IT systems, market mode.

Abstract. The issue of Order No. [2015]9 by CPC Central Committee and State Council, Opinions on Further Deepening Electric Power System Reform, and its supporting documents launch new round of deregulation of electric power industry. According to analysis of the most likely three modes of future China electricity markets and function transformation of system operator, from many aspects such as data quality management, development, operation and maintenance of IT systems, this paper discusses the impacts of electric power system reform on IT systems of system operator in detail. Then, several targeted measures are presented to help system operator to improve the function and performance of its IT systems.
\end{abstract}

\section{Introduction}

In order to implement the decisive role of market on resource configuration, build competitive energy market, and let market determine energy price, CPC Central Committee and State Council issued Order No. [2015]9, Opinions on Further Deepening Electric Power System Reform [1], which presented the general reform idea represented by 'open in three areas, establish independent power exchange and strength government supervision in three areas'. Half a year later, its six supporting documents, which issued by National Development and Reform Commission and National Energy Administration, detailed the direction and content of electric power system reform [2,3].

Peking power exchange and Guangzhou power exchange founded on March, 1st 2016, then many provincial governments established their own power exchanges, launched comprehensive or special pilot electricity market projects, and encouraged load retailer to take part in market competition. Electricity transaction became more active, especially direct trading between generators and industrial customers. The energy traded in market increased rapidly in areas operated by State Grid Cooperation Company(SGCC), which reached 10 percent or more of total consumptions.

With the development of electric power system reform and increasing of energy traded in market, the responsibility and business process of system operator will change dramatically. The traditional dispatching mode named with open, fair and justice, cannot satisfy the operation requirement of electric power system, and will bring a series of challenges on IT systems now in use. Therefore, this paper presents the most likely three modes of future China electricity markets, analyzes the impacts on IT systems of system operator, and presents several feasible targeted measures to guide the upgrade of IT systems to provide strong technical support for stable electric power system reform and secure operation of power grid.

\section{Main modes for future electricity markets}

Market Mode is a general appellation of operation mechanisms of electricity markets, which include definition of electric power commodities, trading cycles, trading styles, pricing mechanisms, information publishing and responsibilities of market participants. Selection of market mode has strong relationship with national energy development strategy, generation structure, power grid structure, load structure, and will change along with the development of electric power industry. 
The following three market modes will be the selection of China electricity markets in the future electric power system reform. The first market mode is direct energy trading and similar contract progress scheduling. In the electricity markets adopting this market mode, energy will be the most popular commodity and only traded in mid-term and long term electricity markets, such as year ahead or month ahead markets, and planning mechanism still dominates in day ahead and real time operation. In order to fulfill the long term transactions signed between market participants by similar progress, system operator schedules according to the energy contract and cumulative energy generated. And establishes ancillary service sharing mechanism by principle of who benefits and who pays. The second market mode is mid-term and long term physical energy markets pricing at specified time interval, such as one hour or fifteen minutes, and day ahead and real time imbalance energy markets. In the long term electricity markets, generators and consumers trade directly to achieve agreements, and provide day ahead generation schedule and consumption schedule before the gate closure time, generally one day before delivery. In this mode, day ahead and real time imbalance energy markets are established to clear operation schedule of generations according to their offer and bid information for imbalance market. At the same time, long term ancillary service markets are established, which permit market participants to trade bilaterally or through power exchange. System operator should dispatch ancillary service in real time operation according to market outcome. This mode is also called decentralized electricity market mode in the supporting document of Order. [2015]9. The third market mode is financial electricity markets pricing at specified time interval, such as one hour or fifteen minutes, and spot electricity markets, which include day ahead electricity markets and real time electricity markets. In the mid-term and long term electricity markets, even to the date before day ahead market, generators and consumers trade directly to achieve financial agreements, and market participants are option to provide day ahead generation schedule and consumption schedule to power exchange for settlement and billing. In day ahead and real time markets, market participants should offer or bid at full capacity according to market rules. System operator clears day ahead and real time markets to minimize total production cost of energy and ancillary service by considering technical constraints of generation, constraints of balance between supply and consumption, and security constraints of transmission devices. System operator should dispatch in real time according to spot markets outcome. This mode is also called centralized electricity market mode in the supporting document of Order. [2015]9.

It is a systematic project to establish electricity markets. Deregulation of electric power industry will impact the entire electric power supply chain of generation, transmission, distribution and consumption, will propose a revolutionary change on existed management system and business process, and will also bring great challenge on existed technical support systems. It is time consuming to amend related law and policy, design electricity markets infrastructure, draw up market rules, and to develop market operation system. According to experience, the establishment of spot electricity markets will cost about two years or more. Therefore, it will cost more time to establish electricity markets with complete trading cycles, rich types of electric commodities and multiple trading styles. It is foreseeable that the market mode of direct energy trading and similar contract progress schedule will still be popular in the next two years, and the scale of trading energy will enlarge rapidly. With increasing energy traded in markets and building of short term electricity marke, the equilibrium between trading energy and planning energy changes fundamentally. More challenges apperar in opeartion with market mode of direct energy trading and similar contract progress schedule.

Market mode 2 and mode 3 should be selected for future electricity markets. However, market mode 3 will be the best choice for future electricity markets for its flexible market mechanism, suitability for all electric power grid structure, supporting locational marginal pricie mechanism, co-optimization of energy and ancillary services, and be conductive to achieve seamless integration between security operation of electric power grid and economic operation of electricity markets. 


\section{Responsibility of system operator under electricity markets}

Under existing operation mechanism, system operator optimizes operation schedule to guarantee secure operation of electric power grid according to yearly generation contracts authorized by government, inter-provincial and inter-regional transaction agreements between market participants, direct transaction between generators and consumers. The responsibilities of system operator mainly include monitoring and control of grid operation, analysis of transmission capability, optimization of unit commitment and output schedule, optimization of tie line schedule, optimization of outage schedule, decomposition of energy contract, integration of renewable generation, simultaneous feasibility test (SFT) of transaction and congestion management.

Under electricity markets, the following new responsibilities will be added to system operator. (1) Preparation of market rules of spot electricity markets. (2) Building and maintenancing of spot market operation system. (3) Verification of registration parameters of market participant related with spot markets. (4) Organizing transaction, publishing information, accepting offer and bid, and clearing of spot markets. (5) Implementation of operation schedule, etc.

\section{Impacts on IT systems of system operator}

In deregulation process of electric power industry, the balance between responsibilities and available resources of system operator may be broken. Mismatch of responsibilities and available resources could lead to capability degradation to guarantee the secure, high quality and economic operation of electric power grid, and brings new challenges on existing technical support systems. In order to improve grid operation performance, SGCC and China Southern Power Grid(CSG) both pay great attention on research of key technology of smart grid operation in recent years, and have developed smart grid operation support system(SG-OSS) [6,7] and integrated operation support system(OS2) [8]. Although requirements of electricity markets have been considered to some degree in design of software platform [9], the deregulation of electric power industry will bring great impacts on grid operation, normalized dispatch and integration of renewable generation. The existing IT systems could not satisfy the requirements of monitoring, analysis and control of electric power grid under electricity markets, and the long term electricity market operation system based on SG-UAP platform could not satisfy the operation requirements of spot markets either [10-12].

\section{impact on life cycle of IT systems}

Under electricity markets, transmission company should minimize the total cost of transmission devices in the premise of reliable and high quality of power supply. Too much attention is focused on minimized acquisition cost of IT systems in traditional resource management mechanism, and less attention is put on maintenance cost and disposal cost. According to the calculation rules of transmission and distribution tariff, the cost of IT systems should be considered from lifecycle view. Therefore, system operator should focus on asset lifecycle management to maximize return of investment by coordination among efficiency, cost and risk of IT systems.

\section{Impact on technical support capability of transmission capability calculation}

Transmission capacity is the dominant factor in power resource configuration in large area. As it could influence the relationship between supply and consumption, transmission capability calculation and available transmission capability calculation play important role in electricity market operation. Accurate and timely transmission capability calculation could maximize potentiality of resource configuration of electric power grid, and provides helpful information for market participants to make rational offer and bid decision. Transmission capability changes with variation of status of generation and transmission device, and available transmission capability also change along with transaction agreements between market participants [13]. Now, transmission capability is updated yearly or monthly and could not reflect actual transmission capability in day ahead and real time electricity markets. Transmission capability could not be utilized sufficiently.

System operator always selects monitoring element list, contingency list and network topology by experience in existing transmission capability calculation, and carry out offline power flow 
calculation, contingency analysis by BPA software. Then, system operator changes monitoring element status, calculates power transfer distribution factor, and tries many predetermined methods to calculate transmission capability. The process of transmission capability calculation is very complex and tedious. Accuracy of result has great relationship with work experience of system operator. With the development of spot electricity markets, the requirement of transmission capability calculation daily will appear, and most important information about transmission capability should be published publicly. It need more effective calculation method and upgrading of existing software.

\section{Impact on technical support capability of SFT}

SFT, the most important measure of control center to guarantee secure operation of electric power gird under electricity markets, will directly influence the benefit of market participants. Now, multi-period SFT software for day ahead and real time generation schedule has been developed and widely used by most system operator of SGCC. However, the following problems still make it unsuitable for market operation.

(1) The function of SFT still need to be extended. SFT should not only consider secure operation, but should also consider many other factors, such as similar contract progress and integration of renewable generation.

(2) The level of refinement of SFT should be improved. Unbalanced distribution of transaction between market participants might deteriorate existed congestions of network or create new congestions. Existing long term transaction SFT is experienced based, and it does not satisfy future requirements. We should reference the method used in day ahead generation schedule optimization, and transform the long term SFT from experience based method to quantitative analysis method. Then, the accuracy and standardization level of long term transaction SFT will be improved remarkably.

\section{Impact on smart grid operation support system}

The type of market members increases in new round of electric power system reform. Each type of market members, such as generator, consumer, load serving entity, system operator, power exchange, market regulator, play important market role. As requirement, such as integrity, reliability, consistency, timeliness, on market data become more rigid, it will be more complex to exchange data among market participants or market applications [14]. Existing smart grid operation system is not designed fort electricity market operation, and cannot support spot markets without upgrading. In order to provide technical support for operation of day ahead market, real time market and ancillary market, it is of great value to improve the design of smart grid operation system.

\section{Impact on requirement of data quality}

As the volume of data created by the main application, which include bid data processing, transaction management, day ahead market, real time market, ancillary market, information publishing, settlement and billing, etc., increases rapidly compared with traditional operation mode, the data processing and management capability of existing technic support systems could not meet the requirement of electricity markets. Thus, the data management system should be promoted to support data acquisition, storage, reading, application and maintainence. For great volume and high frequency of data change between applications, high efficient and universal data exchange function should be designed and developed to improve data processing capability and guarantee the integrity, reliability, consistency and timeliness of operation data in electricity markets.

\section{Impact on cybersecurity protection}

The users of existing smart grid operation systems are mainly system operator. Condition will change as generator, consumer, load serving entity, power exchange and market regulator become new users. As many users are not familiar with cybersecurity protection scheme of electric power system, and cannot afford the cost to build similar cybersecurity infrastructure as system operator or generator, new cybersecurity infrastructure should be established. 


\section{Main countermeasures}

Through the analysis of future market mode and its impacts, we know that the market mode of direct energy trading and similar contract progress schedule still be the most popular market mode in the next two or more years. In spite of the reduction of yearly energy contract authorized by government, energy contract authorized by government and transaction contract will coexist for a long time. All the energy contracts are executed by system operator according to principle of similar progress. Schedule optimization of day ahead and real time operation lacks consistent standard and system operator will face great challenge to coordinate the secure and economic operation of electric power grid. With the development of deregulation of electric power industry, relative perfect electriccity market mechanism will be established, and new balance between responsibilities and available resources of system operator will be achieved. In order to accommodate reform of electric power system and improve technical support capability, the following measures should be taken.

\section{Improving management and calculation of available transmission capability}

In order to meet the requirement of establishment and operation of spot electricity markets, the management and calculation of transmission capability, available transmission capability should be improved in following aspects.

Firstly, the requirement of each business department should be considered in the calculation of transmission capability, and the data used should be the same of Energy Management System to guarantee the consistency and timeliness of basic information. Secondly, the calculation process should be open to relevant market participants, who can get all the data used in calculation, such as network model, boundary condition, thermal limit of transmission device, and could reproduce each step of calculation to verify the calculation of transmission capability. Thirdly, integrated network model, real time power flow data, typical operation data, model of automatic security device, thermal transmission limit should be maintained, all the base state transmission constraints, $\mathrm{n}$ minus one constraints should be considered in calculation, and calculation result should be verified and audited. Fourthly, the design of hardware and software of transmission capability calculation platform should be promoted to improve the calculation efficiency. Then, the transmission capability could be calculated daily to meet the requirement of spot electricity markets. Finally, the available transmission capability between some important buses should be calculated dynamically to provide essential information for market participants to carry out new transaction. Once upon the market be cleared or bilateral transaction result be provided, all the available transmission capability between the important buses should be recalculated and published simultaneously.

\section{Improving quality and management of basic data}

Because operation data of electricity markets is published frequently and less time is left to system operator to verify and audit, the quality and management of basic data should be improved substantially.

In data acquisition process, we should promote performance of data acquisition devices, establish comprehensive management process of data input manually, normalize data biding. In data verification process, we should identify bad data via state estimation or data mining technology, rectify bad data by automatic data revising technology. In data model building process, we should establish model verification mechanism, promote data updating mechanism via multi-database backup, and analyze integrity, reliability, consistency, timeliness, accuracy of operation data via establishing data quality evaluating mechanism. Lots of optimization could be done in database design, database management, and application design to improve data process efficiency. In database design level, it is of great value to adopt multi-thread processing, parallel computing, reading strategy optimization, use network computation, memory buff pool and data compressing technology to promote data reading efficiency. In database management level, data reading efficiency could be improved by adopting unified interface between database and applications, reducing resource cost of database sessions. In application level, data processing efficiency could be improved by creating calculation column, reducing number of unnecessary row and column, optimizing data reading and task processing sentences, avoiding database dead lock. 


\section{Improving technical support capability for spot electricity market operation}

In order to meet requirement of spot electricity market operation, the following measurements should be taken to promote technical support capability on the basis of existing smart grid operation support system.

Firstly, we should present more robust infrastructure design of electricity market operation system according to main business processes taken by system operator, and lay a solid foundation for development and application of future electricity market operation system. Secondly, we should expand existing network model to take economic relationship among market participants into integrated management, build relationship between transaction and generators or loads, and to support time traceable market model management by referring international standard IEC 62325-301, Common Information Model(CIM) of electricity market. Thirdly, we should develop mid-term and long term SFT software for yearly and monthly direct transaction between generators and consumers, which could carry out simultaneous feasibility test on market result automatically and present congestion mitigation advices by considering load forecast, transmission and generation outage schedule, network topology, and existed transaction. Finally, we should develop security constrained optimization software for day ahead and real time electricity markets, which clear electricity market with minimized total production cost, develop optimization software which could optimize energy and ancillary services together according to market mode, research high performance data biding and reading technologies for vast amount market participants access, and should research cybersecurity scheme which be fitted for electricity market operation.

\section{Establishing cybersecurity system for electricity market operation}

We should establish cybersecurity system for electricity market operation to promote capability of security protection. Based on existing security infrastructure, we could establish integrated cybersecurity protection system, and build disaster recovery mechanism by giving consideration to efficiency and cost. For critical business or information, we should take multi-level protection measures and build behavior audit system to find out abnormal behavior as soon as possible. At the same time, we should strength application of data encryption, establish fast communication channel via optimizing security devices, popularize application of GMB algorithm, and build multi-layer data backup mechanism to keep data securely.

\section{Conclusions}

Subject to the constraints of generation structure, power grid structure, load structure, laws and regulations, knowledge structure of employee of China electric power industry, it will be a long term course to establish comprehensive electricity markets. Mid-term and long term energy transaction will still popularize in the forecastable future, and similar contract progress of mid-term and long term transaction will still be the most important task of system operator. Hence, the market mode of direct energy trading and similar contract progress schedule will popularize in the transitional stage. Since control on dispatchable resources is weakening, the ability to reliably perform their duties of system operator, which relies on existing technical support system without fundamental improvement, will weaken too. Based on detail analysis of the impacts on system operator, several advices, which include improving management and calculation of available transmission capability, improving quality and management of basic data, improving technical support capability for spot electricity market operation, and establishing cybersecurity system for electricity market operation, are presented to provide strong technical support for stable reform of electric power system and secure operation of power grid.

\section{Acknowledgements}

This work was financially supported by the State Grid Cooperation Company Science and Technology Foundation ((DZN17201500036). 


\section{References}

[1] CPC Central Committee and State Council, Opinions on Further Deepening Electric Power System Reform, Order.[2015]9, Mar 15, 2015. In Chinese.

[2] National Development and Reform Commission and National Energy Administration, Order.[2015]2752,Opinions on Promoting the Construction of Electricity Market, Nov 29,2015. In Chinese.

[3] National Development and Reform Commission and National Energy Administration, Order.[2015]2752,Opinions on the Implementation of Standardization and Operation of Power Exchange, Nov 29,2015. In Chinese.

[4] ZHANG Xian,LIU Fubin,PENG Tao, et al.A Discussion on Key Issues for Direct Trading Between Power Users and Plants[J].Automation of Electric Power Systems,2014,38(13):33-37.

[5] XIA Qing.Institutional Design and Suggestions for Promotion of Direct Electricity Purchase by Large Consumers in China[J].Automation of Electric Power Systems,2013,37(20):1-7.

[6] XIN Yaozhong,SHI Junjie,ZHOU Jingyang, et al.Technology Development Trends of Smart Grid Dispatching and Control Systems[J].Automation of Electric Power Systems,2015,39(1):2-8.

[7] GAO Kunlun,XIN Yaozhong,LI Zhao, et al.Development and Process of Cybersecurity Protection Architecture for Smart Grid Dispatching and Control Systems[J].Automation of Electric Power Systems,2015,39(1):48-52.

[8] WANG Jifeng.Concept and Features of Integrated Grid Operation Smart System[J].Automation of Electric Power Systems,2011,35(24):1-6.

[9] DING Qia, LI Lili, TU Mengfu et al. Design and Key Technologies of Day-ahead Generation Scheduling System for Smart Grid [J]. Power System and Clean Energy, 2013,29(9):1-5.

[10]ZHANG Xian,ZHENG Yaxian,GENG Jian, et al.Design of Direct Trading Platform for Electricity Users and Power Generation Enterprises Supporting Whole Business Procedure[J].Automation of Electric Power Systems,2016,40(3):122-128.

[11]SHANG Jincheng, ZHANG Xian, GAO Chuncheng et al. Business Analysis and Design of Platform for Direct Transactions Between Large Power Consumers and Generation Enterprises[J]. Power System Technology, 2011,35(9):199-204.

[12]ZHOU Sheng, TAO Min, XU Peng et al. Study on Universe Access Method of Real-time/historical Data Platform Based on SG-UAP [J]. Zhejiang Electric Power,2015,35(4):70-73.

[13]LYU Ying,LU Guangming,YANG Junfeng, et al.Security Check Service and Practical Technique Based on Smart Grid Dispatching and Control Systems[J].Automation of Electric Power Systems,2015,39(1):171-176.

[14] Ott, A.L.: Experience with PJM market operation, system design, and implementation. IEEE Trans. Power Systems 18(2), 528-534. 